\title{
Precise In-Hand Manipulation of Soft Objects using Soft Fingertips with Tactile Sensing and Active Deformation
}

\author{
Qiujie Lu*, Student Member, IEEE, Liang He*, Student Member, IEEE, \\ Thrishantha Nanayakkara, Senior Member, IEEE, and Nicolas Rojas, Member, IEEE.
}

\begin{abstract}
While soft fingertips have shown significant development for grasping tasks, its ability to facilitate the manipulation of objects within the hand is still limited. Thanks to elasticity, soft fingertips enhance the ability to grasp soft objects. However, the in-hand manipulation of these objects has proved to be challenging, with both soft fingertips and traditional designs, as the control of coordinated fine fingertip motions and uncertainties for soft materials are intricate. This paper presents a novel technique for in-hand manipulating soft objects with precision. The approach is based on enhancing the dexterity of robot hands via soft fingertips with tactile sensing and active shape changing; such that pressurized air cavities act as soft tactile sensors to provide closed loop control of fingertip position and avoid object's damage, and pneumatic-tuned positive-pressure deformations act as a localized soft gripper to perform additional translations and rotations. We model the deformation of the soft fingertips to predict the in-hand manipulation of soft objects and experimentally demonstrate the resulting in-hand manipulation capabilities of a gripper of limited dexterity with an algorithm based on the proposed dual abilities. Results show that the introduced approach can ease and enhance the prehensile inhand translation and rotation of soft objects for precision tasks across the hand workspace, without damage.
\end{abstract}

\section{INTRODUCTION}

Precise in-hand manipulation is an important and essential ability for robot grippers to achieve small-scale adjustive manipulation tasks without unnecessary large arm motions. Recent research in dexterous in-hand manipulation has focused on achieving particular tasks or performing certain movements of a rigid object, for example reorienting the object [1], [2] or performing a prehensile spherical motion [3]. However, the development of robotic grippers that can precisely in-hand adjust soft objects has received limited attention. For grasping soft objects, soft fingertips with tactile sensors perform better than rigid fingertips due to their high grasping compliance. With rigid fingertips, robotic grippers can achieve a repeatable stable grasping force and high precision control with the ease of integrating high standard sensors and control algorithms [4], [5]. Nevertheless, rigid fingertips lack adaptability and compliance, and they are prone to damage and may deform very fragile and soft objects.

With high compliance of the soft fingertips, the increased contact area with objects results in a greater variety of moments to the grasped object [6]. Most current research focuses

Qiujie Lu and Liang He contributed equally to this paper. Authors are with Dyson School of Design Engineering, Imperial College London, 25 Exhibition Road, London SW7 2DB, UK. (e-mail: \{q.lu17, 1.he17, t.nanayakkara, n.rojas\}eimperial.ac.uk)

This work was supported in part by the Engineering and Physical Sciences Research Council grant EP/R020833/1.

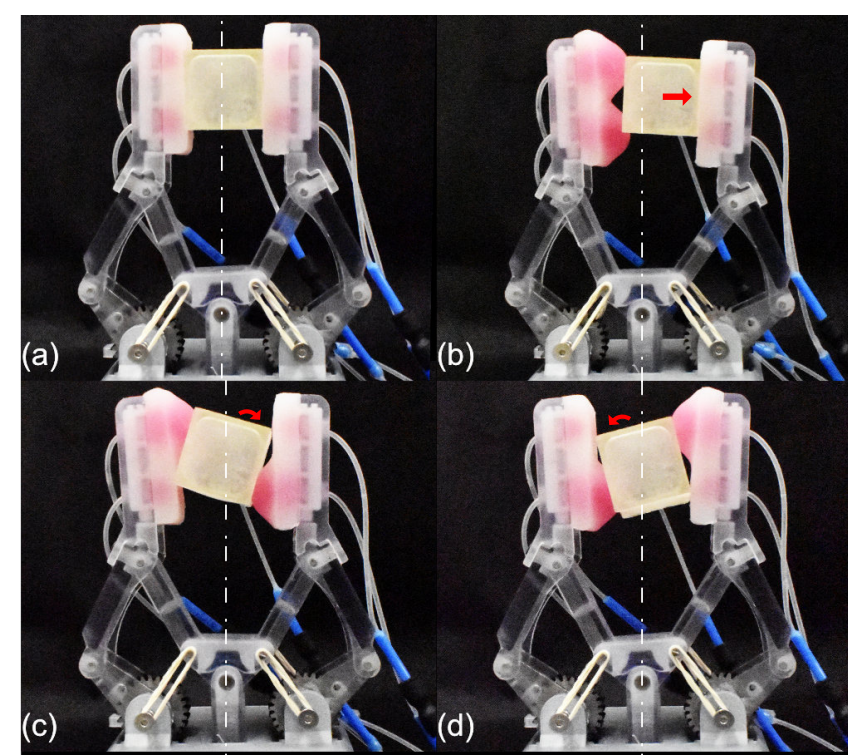

Fig. 1. The ability of robot hands to manipulate soft objects with precision can be enhanced using soft fingertips with adaptive sensing and active shape changing (a). Embedded air cavities in these soft fingertips allow the dual purpose of soft tactile sensing, via internal pressure variation, and active inhand translation (b) and rotation (c, d), via positive pressure. White dotted lines indicate the center line of the gripper. Red arrows indicate the object's motion (semi-transparent square)

on delicate grasping by robotic grippers. For instance, Amend et. al proposed the well-known granular jamming universal gripper for grasping a wide range of objects [7]; Maruyama et. al presented a gripper with incompressible fluid covered by rubber fingertips which can grasp fragile and brittle objects by controlling the contact pressure [8]; and Manti et. al showed the dexterous grasping capability with a simple control of a bioinspired soft gripper [9]. However, these gripper and fingertip designs do not have the capability of in-hand and precision manipulation.

In-hand manipulation of soft objects with a simple degree of freedom gripper is not well explored as yet. The main difficulties are the actuation method, force control, and the soft fingertip model. The dexterity of robotic grippers can be increased by changing the friction of the fingertip [10], [11] or by applying external forces [2]; however, these approaches are not suitable for soft objects. Tactile sensors are commonly being employed on the fingertip to sense the grasping force [5] and measure the object surface texture and shape [12], but it is difficult to perform active deformation with those conventional tactile sensors. The relationship between soft fingertips and 


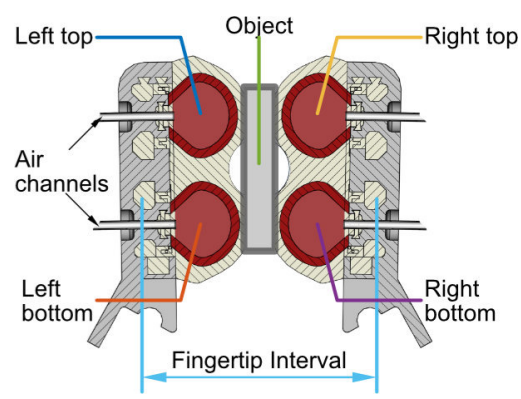

(a)
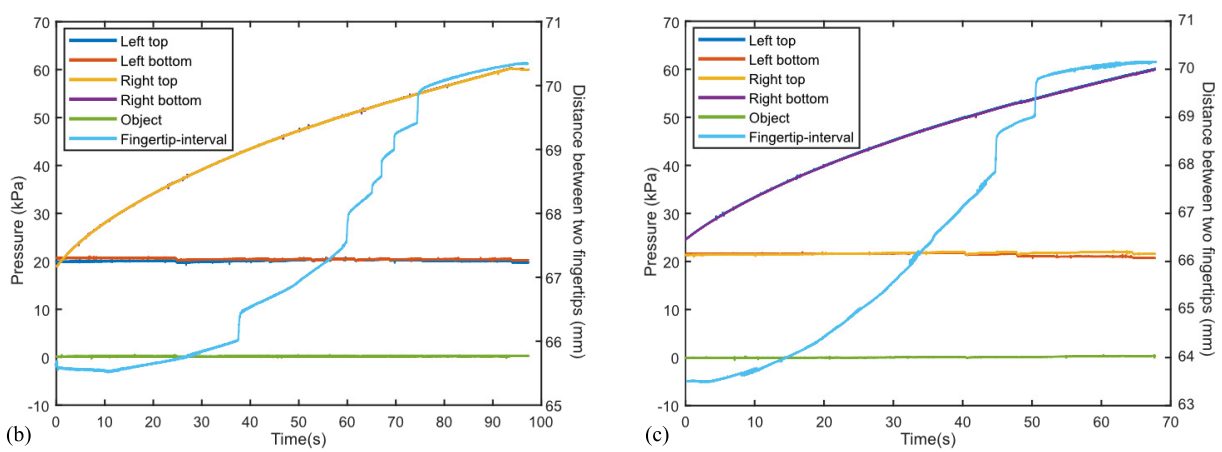

Fig. 2. (a): Schematic of the dual function fingertip design. The line graphs show the internal pressure data of four air cavities and soft object manipulated (green), and distance between two fingertips (light blue) (recorded by motion tracking cameras) for a translation task (b) and a rotation task (c).

in-hand manipulation is not well defined as well. Due to the uncertainties of soft materials (e.g., rolling, deformation), it is hard to model the exact manipulation of the grippers and the dynamic motion of the soft objects [13], [14].

In this paper we propose a novel soft fingertip design for in-hand manipulating soft objects via embedded air cavities (1). This fingertip has dual purpose of tactile sensing and active deformation which allows the GR2 gripper [15] to robustly grasp [16] and in-hand manipulate soft objects. Soft objects can be displaced within the gripper to a certain extent only by controlling the shape of fingertip air cavities. The tactile pressure sensing in the fingertip air cavities can be used for closed loop control of contact force during object manipulation.

In the rest of the paper, we detail the fingertip design and the control algorithm for soft objects (section II); characterize and model the deformation of the soft fingertip to predict the motion of the objects (section III); evaluate in-hand manipulation (translation and rotation) performance of the developed dual purpose soft fingertips with a soft object (section IV); and discuss the simulation and gripper performance in section $\mathrm{V}$ and conclude in section VI.

\section{FInGertip CHARACTERIZATION \& CONTROL}

\section{A. Fingertip \& gripper design}

Figure 2(a) shows the schematic of the dual fingertip design holding a soft square object, where the two embedded air cavities (red sections) are evenly distributed in each fingertip made from silicone rubber (white sections). The special fingerbase structure is 3D printed by Objet 260 in VeroClear, which is designed to secure the silicone fingertip and the air, and ensure the inflate direction is growing as desire. Both the soft fingertips and the half-cylindrical air cavities are made from the same silicone material (Ecoflex-30). The shore hardness of the fingertip is around $25 \mathrm{HC}$ with the material and around 20 on the cavity at $0 \mathrm{kPa}$ (relative to atmospheric pressure), measured by a type $\mathrm{C}$ durometer. Here, the soft square object is also 3D printed by Objet 260 but with a soft material (FLX 9960-DM). The proposed fingertip is attached to the fingers of a redesigned GR2 gripper [15] (Fig. 1) to perform in-hand manipulation tasks. Instead of tendon driven actuation, the

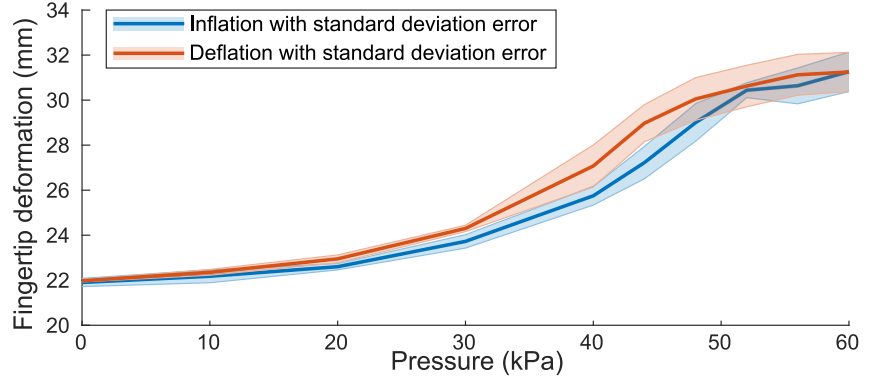

Fig. 3. Fingertip characterization: fingertip maximum deformation variation of inflation (blue) and deflation (red).

TABLE I

DUAL FINGERTIP PRESSURE CHARACTERIZATION

\begin{tabular}{l|l|l|l|l|l|l|l|l|l|l}
\hline \hline Pressure $P$ (kPa) & 0 & 10 & 20 & 30 & 40 & 44 & 48 & 52 & 56 & 60 \\
\hline$D_{\max }(\mathbf{m m})$ & 21.9 & 22.3 & 22.8 & 24.1 & 26.4 & 28.1 & 29.5 & 30.5 & 30.9 & 31.3 \\
\hline \hline
\end{tabular}

implemented gripper uses a gear mechanism (KHK 28) to increase manipulation accuracy.

\section{B. Fingertip characterization}

We use the embedded air cavities as soft tactile sensors to control the gripper position to in-hand manipulate soft objects via the internal pressure variation. Fig. 3 shows the fingertip deformation with pressure, when the air cavity is inflated, the deformation of the air cavity causes shape change of the fingertip. In order to characterize this behaviour, the fingertip deformation value is measured by a caliper at the widest part of the fingertip. First the baseline bias due to atmospheric pressure is removed. Then the biased-removed internal pressure can rise to a maximum of $60 \mathrm{kPa}$ during inflation. The curves in Fig. 3 represent the average data of 5 trials of inflation/deflation tests. A detailed hysteresis and sensitivity analysis can be found in [16].

\section{Control algorithm}

The four soft air cavities and the soft square object (i.e., the red bubbles and the semi-transparent object in Fig. 2(a), respectively) were connected with 5 pressure sensors (PSE 


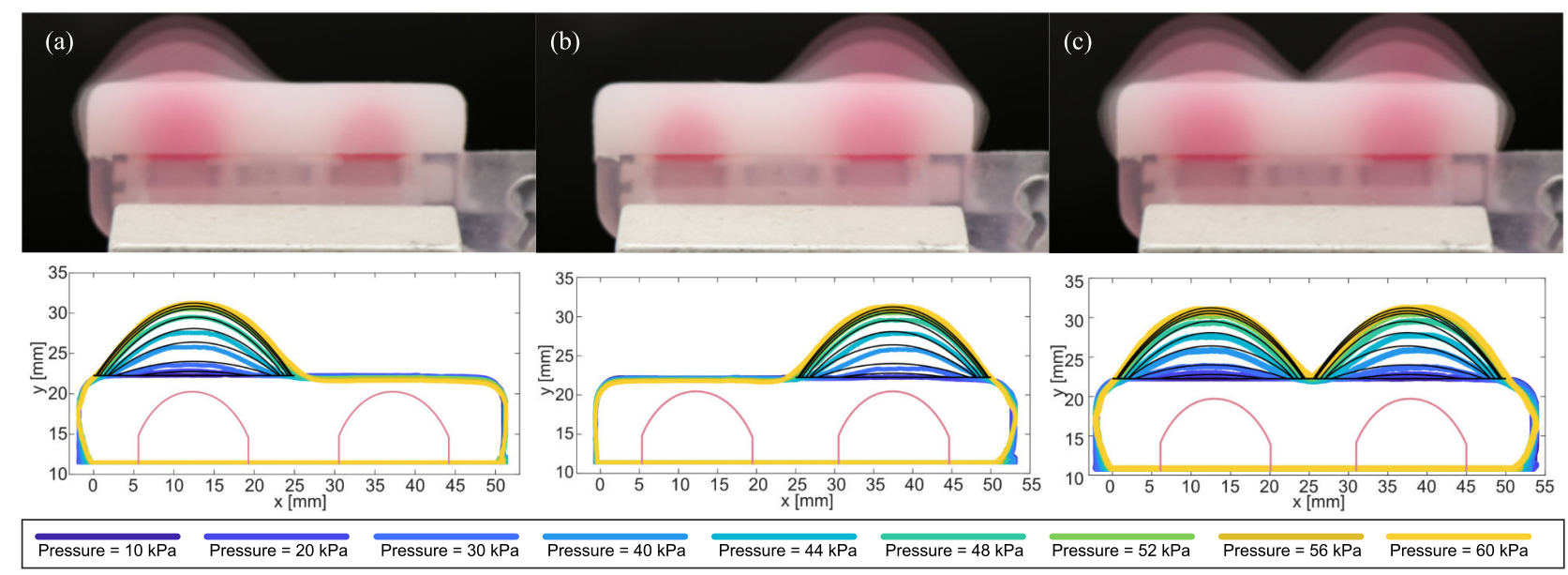

Fig. 4. Numerical deformation of the fingertip based on image processing of a real inflated and deflated fingertip on various pressure levels.

543-R06) and 5 solenoid valves (Z031C), one air reservoir, and an air pump. Labview 2018 was used to measure the internal air pressure via a National Instrument DAQ (USB-6341) and control the gripper servo via a U2D2 USB communication converter. For both translation and rotation, the initial grasping air pressure of the fingertip is $20 \mathrm{kPa}$. The aim is to in-hand manipulate the soft object without over increasing the object internal air pressure via active sensing and active deformation. The control algorithm for in-hand translation and rotation is described below.

1) Translation: As demonstrated in Fig. 1(b), both air cavities of left fingertip are inflated for moving-right translation tasks. Instead, both air cavities of right fingertip are inflated for opposite translation direction. The rest of the fingertip is acting as an active tactile sensor to ensure the contact forces between fingertips and soft object are constant during manipulation by actuating the servo motors. Fig. 2(b) shows the internal pressure reading of all air cavities and the object. When righttop and right-bottom air cavities are inflated from $20 \mathrm{kPa}$ to $60 \mathrm{kPa}$ (active deformation), the left top and bottom air cavities are set to keep the pressure under $22 \mathrm{kPa}$ to ensure the object internal pressure reading is steady (green). The gripper position is then controlled by the air cavities automatically, in this case, if either the left-top or left-bottom air cavities' internal pressure is higher than $22 \mathrm{kPa}$, then the left motor opens 1 degree more until the pressure is under $22 \mathrm{kPa}$.

2) Rotation: Figure 1(c) and (d) illustrate the in-hand rotation of the soft object. When the left-top and rightbottom air cavities are inflated together, the grasped object rotates clockwise. Instead, if the left-bottom and right-top air cavities are inflated together, the grasped object rotates anticlockwise. Fig. 2(c) shows the internal pressure reading during a clockwise rotation task. This time, only one air cavity of a single fingertip is inflated, so both fingertips have the active tactile sensor on, and the gripper can open in both directions. When the left-top and right-bottom air cavities are inflated from $20 \mathrm{kPa}$ to $60 \mathrm{kPa}$, if either the left-bottom or right-top air cavities' internal pressure is higher than $22 \mathrm{kPa}$, then the corresponding motor opens 1 degree more until the pressure is under $22 \mathrm{kPa}$. The light blue curves illustrate the distance changes between two fingertips during the manipulation.

\section{Simulation MODEL}

The proposed simulation models the deformation of the soft fingertips to predict the in-hand manipulation of manipulated objects.

\section{A. Method}

To characterize how the fingertip deforms when different pressure conditions are applied to the air cavities, image processing was used to extract the planar fingertips outlines. The fingertips were placed in front of a camera where a set of pictures were captured corresponding to different internal air pressure conditions. Image data were processed with MATLAB Image Processing Toolbox. Each air cavity is assumed to be symmetric with its peak lying on the center line. The vertical coordinate $y$ of the peak point at the pressure condition $p$ is assumed equal to the characterized fingertip width $D_{\max }(p)$. Second order polynomial functions were used to fit the outlines of the fingertips. The two air cavities are assumed to be independent during inflation and interference between the two inflated air cavities are neglected. Fig. 4 shows the images used for analysis and the fitted models with pre-characterized parameters.

\section{B. Model}

The boundary of the fingertip based on Fig. 4 is described by the following equation

$$
y(x)=\left\{\begin{array}{ll}
K(p)(x-12.5)^{2}+D_{\max }(p) & \text { if } 0 \leq x<25 \\
K(p)(x-37.5)^{2}+D_{\max }(p) & \text { if } 25 \leq x \leq 50
\end{array},\right.
$$

where the origin is located at the top corner of the fingertip base, $x$ is the width of the fingertip and $y$ is the thickness of the fingertip. Both $K$ and $D_{\max }$ are pressure related variables of the equations, such that $K(p)=0.082 p^{3}-8.3 p^{2}+99.5 p+$ $1.387 \times 10^{-3}$ and $D_{\max }(p)=0.025 p^{2}+0.0244 p+21.6897$, 


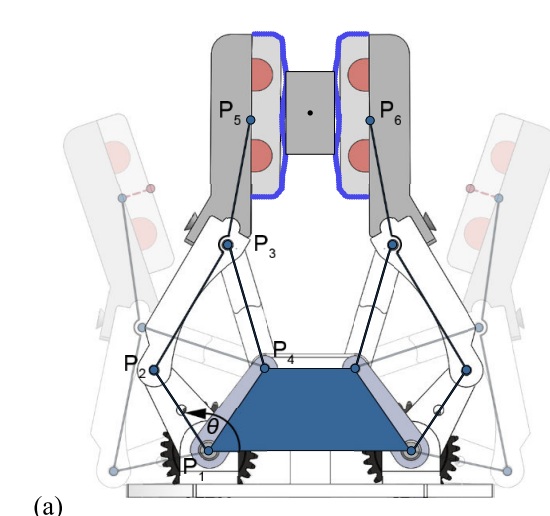

(a)
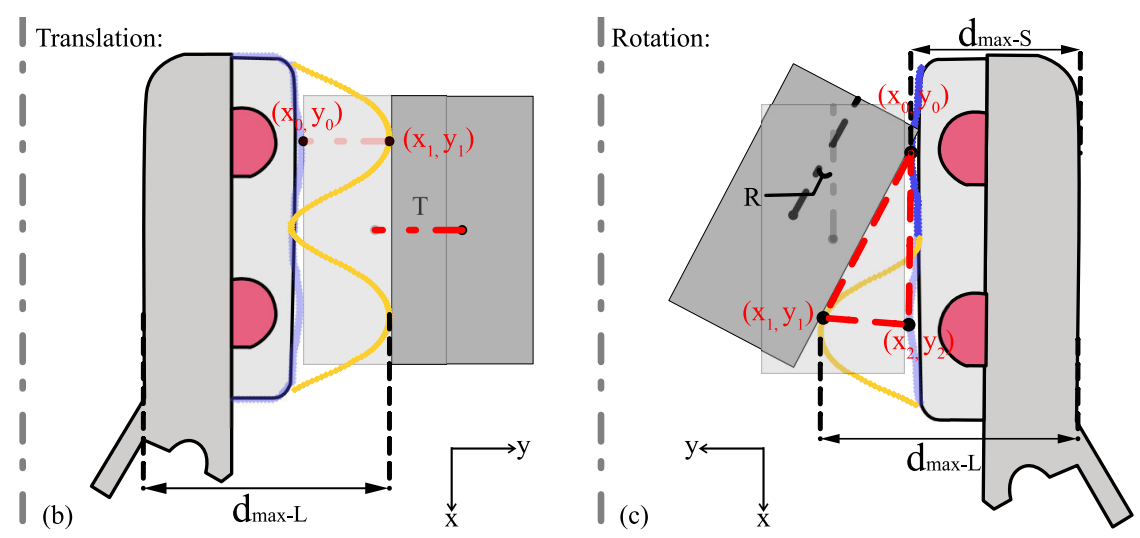

Fig. 5. Simulation model illustration. (a): Kinematic chain of the gripper. $P_{1}, P_{2}, P_{3}, P_{4}$ are revolute joints on the left gripper. $P_{5}$ and $P_{6}$ determine the fingertip interval distance. $\theta$ is the motor input actuation angle. (b): Translation task model: the faded blue boundary indicates the initial grasping position $\left(x_{0}, y_{0}\right)$ and the yellow boundary indicates the fingertip inflated position $\left(x_{1}, y_{1} 0\right)$. The translation distance is $T$. (c): Rotation task model the contact point $\left(x_{1}, y_{1}\right)$ is the tangent point on the inflated boundary curve that passes through point $\left(x_{0}, y_{0}\right)$. The rotation angle is $R$.

where $P$ is the instant pressure. The expression of $D_{\max }$ is poly-fitted from Table I and $K(p)$ is poly-fitted of the quadratic coefficients of these second order polynomial outline functions.

Based on the control algorithm, the simulation models of translation and rotation task are illustrated in Fig. 5(b) and (c). The figure only illustrates half of the gripper to facilitate explanation. In this simulation, we assume the grasping force is minimal where the fingertip has no passive deformation during the manipulation. The object translation distance $T$ is determined from the object center point distance between the initial stage and final stage where the variable is the pressure level $p$. It can also be expressed as the active inflated deformation $T^{2}=\left(x_{1}-x_{0}\right)^{2}+\left(y_{1}-y_{0}\right)^{2}$, where $\left(x_{0}, y_{0}\right)$ and $\left(x_{1}, y_{1}\right)$ are the coordinates of the contact point at initial and final stage, respectively. We assume the contact point is always at the center of the air cavity, in this case $x_{1}=x_{0}$, so this equation can be simplified as $T=\left|y_{1}-y_{0}\right|$.

Only one air cavity is inflated to rotate the object for one fingertip, we assume that one of the contact points, $\left(x_{0}, y_{0}\right)$, is always at the center of the uninflated air cavity and the other one is the tangent point $\left(x_{1}, y_{1}\right)$ on the inflated air cavity through the fixed contact point. Then, $\left(x_{1}, y_{1}\right)$ can be computed from the equation of the tangent going through these points and the value for $y_{1}$ from equation (1), such that

$$
\begin{aligned}
y_{1} & =K(p)\left(x_{1}-37.5\right)^{2}+D_{\max }(p), \\
\frac{\left(y_{0}-y_{1}\right)}{\left(x_{0}-x_{1}\right)} & =2 K(p) x_{1}-75 K(p) .
\end{aligned}
$$

By replacing equation (2) into (3), we obtain a quadratic equation in terms of $x_{1}$.

All of the three points of the dotted red triangle in Fig. 5, namely $\left(x_{0}, y_{0}\right),\left(x_{1}, y_{1}\right),\left(x_{2}, y_{2}\right)$, are known or can be computed. The rotation $R$ of the object can then be calculated as the intersection angle between the initial object boundary and final object boundary. The simulated translation distances $(T)$ and rotation angles $(R)$ are obtained using this simulation model. The dimension of the fingertip is $50 \times 22 \times 32 \mathrm{~mm}$, the pressure range is from $20 \mathrm{kPa}$ to $60 \mathrm{kPa}$. At the middle grasping configuration, the desired translation $(T)$ is $8.5 \mathrm{~mm}$, and rotation $(R)$ is 18 degrees in one direction. At other grasping positions the object has an initial rotation angle (due to gripper design and rolling), the model assumes that the total translation and rotation range does not change in these cases.

\section{IMPLEMENTATION}

\section{A. Experimental setup}

The aim of this experiment is to show the dual purpose soft fingertip can perform precise in-hand manipulation of soft object with a limited dexterity gripper. The testing object is a 3D printed 30mm soft square in material FLX 9960-DM which is airtight and connected to a pressure sensor (Fig. 6(d)). Its internal pressure variation (Fig. 6(e)) is used to exam the capability of this gripper on manipulating soft object. The gripper translated and rotated the soft square at 7 different grasping positions. The trajectories of the testing object were recorded by motion tracking cameras (OptiTrack Flex3) and post processed in MATLAB.

\section{B. Results}

Fig. 6 shows the method of evaluating the gripper performance and the interpretation of Fig. 7. Fig. 6(a,b,and c) illustrate the results in Fig. 7 which the arrows show the object's initial rotation stages due to the gripper design and rolling aspect, the precise translation (pink shaded area) and rotation (green shaded area) due to active fingertip deformation via positive pressure. The main purpose of this experiment is to keep the soft object safe during the precise in-hand manipulation, in this case, maintaining the internal pressure of the soft object at low level. We unbiased the internal pressure at the beginning of each test to keep the initial reading of the internal pressure of the soft object at $0 \mathrm{kPa}$ (relative to atmospheric pressure). Here, based on the control algorithm in section II.C, we consented the internal pressure of the soft object should less than $0.3 \mathrm{kPa}$ without breaking it. Fig. 

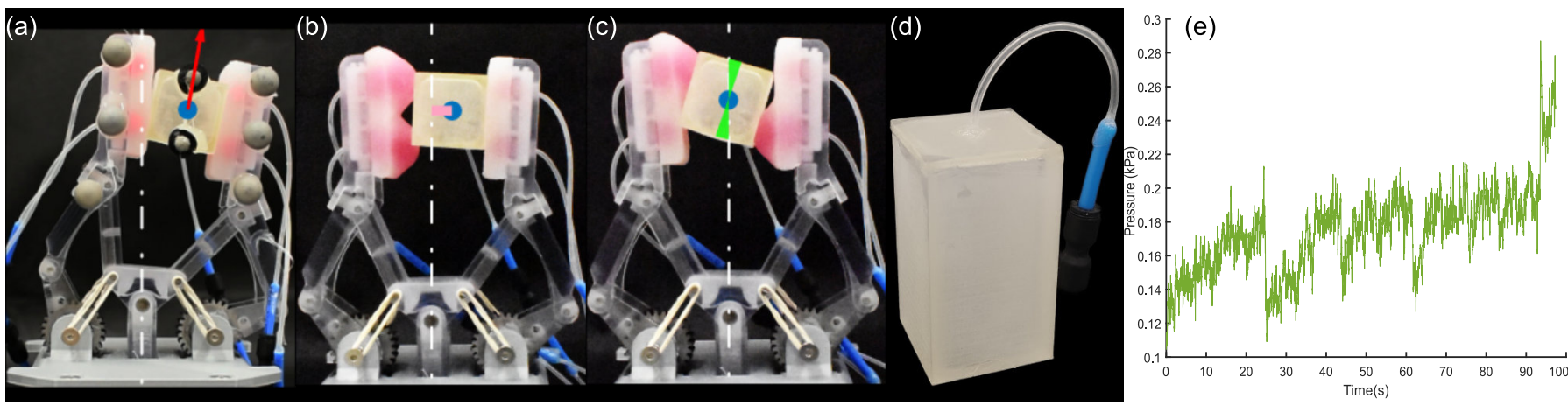

Fig. 6. Illustration of the experiments. Object at grasping position with an initial rotation angle (a), translation (b), and rotation (c). The soft object is shown in (d) and (e) shows the object internal pressure variation during translation task (zoomed-in of the data presented in Fig. 2(b))

6(e) shows a clear pattern of the internal pressure of the testing object which is the same result as Fig. 2(e) in large scale. When the air cavities inflate, the soft object receive external force to have slight deformation, the internal pressure increases. Once the gripper open due to the soft tactile sensing, the internal pressure of the soft object decreases. Those happen repeatedly until the air cavities reach to their limit.

Table II and Fig. 7 present the experimental in-hand translation and rotation capability of this gripper for the $30 \mathrm{~mm}$ soft square at 7 different positions. Those 7 grasping positions are distributed evenly in the GR2 movement range, where position 4 is the middle grasping position, 1 and 7 are the maximum left and right grasping position, respectively (Fig. 6(a) presents the position of 6). Due to the gripper design and the rolling aspect, object had initial rotation angle at each grasping position. Table II shows the results of 5 trials, the initial rotation angles are symmetrical with maximum of 12.09 degrees. The standard deviation of all positions are lower than 1.17 and decreasing towards to the center position. It shows the designed gripper and fingertip are robust for grasping and rolling. The rest of the table summarizes the achievable rotation and translation range of each grasping position. The minimum rotation range is 23.16 degrees at position 1 and the maximum rotation range (29.89 degrees) occurs at position 4 . The translation range at each position has some fluctuation with minimum $(9.45 \mathrm{~mm})$ at position 1 and maximum $(17.24 \mathrm{~mm})$ at position 5 .

\section{DISCUSSION}

The translation and rotation capability of the gripper with the dual purpose fingertips has been shown clearly in Table II and Fig. 7. We observed that the relative position between the fingertip and the object affect the in-hand manipulation performance and both translation and rotation range decrease when the initial rotation angle of the object increase. In a rotation task, if the object has initial rotation stage in the same direction of the target rotation (e.g. the object is held at position 6 and intended to rotate to right), the inflated air cavity will have a gap between the grasping object (Fig. 6(a)). During the inflating stage, the air cavity will touch the object first, and then rotate the object. Therefore, in this case, the object rotation angle is smaller than normal. Conversely, if

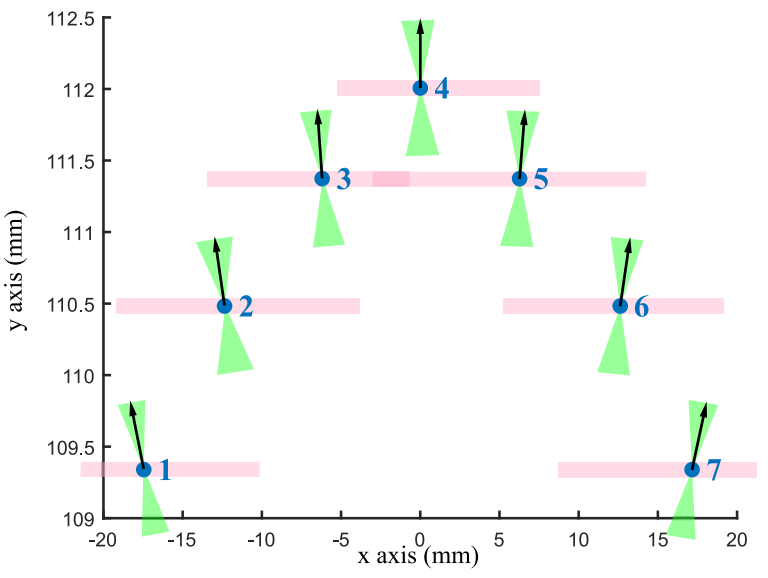

Fig. 7. The 7 different positions evaluated under rotation and translation: blue dots indicate the center point of the $30 \mathrm{~mm}$ soft square. Black arrows show the object initial rotation due to gripper design and rolling. Green and pink shaded area indicates the rotation range and translation range, respectively.

the initial rotation direction and the target rotation angle is opposite (e.g. the object is held at position 6 and intended to rotate to left), the rotation angle will be larger than normal. In Fig. 7 at position 6, it is obvious that the size of the green shaded area on the right side of the arrow is less than the right one. Similar phenomenon appears in translation tasks (pink shaded area) as well.

Although the rotation angle and translation distance in left and right direction are not similar, the total rotation range and translation range of those symmetrical points are similar in Table II. It shows the precise in-hand manipulation capability of the gripper is reliable. There are some variation in translation range, which may occur because the relative position between the fingertip and the object may have slightly differences for each test due to placing the object manually. This may result in different inflating position and sensing position which will affect the sensing capability. The size and shape of the object is also an aspect to consider, if the object does not touch the sensing air cavity properly, the sensing capability will decrease and the feasible manipulation range will decrease as well. Additionally, the testing soft square 
TABLE II

DUAL FINGERTIPS IN-HAND MANIPULATION CAPABILITY

\begin{tabular}{|c|c|c|c|c|c|c|c|c|c|c|c|c|c|c|}
\hline Position No. & \multicolumn{2}{|c|}{1} & \multicolumn{2}{|c|}{2} & \multicolumn{2}{|c|}{3} & \multicolumn{2}{|c|}{4} & \multicolumn{2}{|c|}{5} & \multicolumn{2}{|c|}{6} & \multicolumn{2}{|c|}{7} \\
\hline \multirow{2}{*}{ Init. Rot. Angle ${ }^{\circ}$} & Mean & SD & Mean & SD & Mean & SD & Mean & SD & Mean & SD & Mean & SD & Mean & SD \\
\hline & -11.21 & 1.09 & -8.21 & 0.56 & -3.98 & 0.18 & 0.11 & 0.12 & 4.48 & 0.81 & 8.29 & 0.54 & 12.09 & 1.17 \\
\hline Rot. Range $^{\circ}$ & \multicolumn{2}{|c|}{23.16} & \multicolumn{2}{|c|}{28.08} & \multicolumn{2}{|c|}{29.62} & \multicolumn{2}{|c|}{29.89} & \multicolumn{2}{|c|}{29.31} & \multicolumn{2}{|c|}{27.43} & \multicolumn{2}{|c|}{24.88} \\
\hline Trans. Range mm & \multicolumn{2}{|c|}{11.45} & \multicolumn{2}{|c|}{15.31} & \multicolumn{2}{|c|}{12.76} & \multicolumn{2}{|c|}{12.72} & \multicolumn{2}{|c|}{17.24} & \multicolumn{2}{|c|}{13.87} & \multicolumn{2}{|c|}{12.49} \\
\hline
\end{tabular}

is hollow, the edge of the object is harder than the center, the inflated air cavities have chances to contact either the object edge or softer object body. As the gripper presents better manipulation capability on harder material, where soft material absorb forces and energy during manipulation. When the inflated air cavity touches to the soft object, if the object is softer than the air cavity, the object may deform at the contact point first and then transfer the force to the rest of the object. Nonetheless, results show that the approach enlarges the dexterity of simple grippers and the information about grasping state can be obtained by other means (e.g., vision). The air cavity tactile sensors show acceptable sensitivity for soft objects, increasing the sensing area and sensor number may improve the manipulation performance of soft objects.

Simulation shows the gripper with the novel design fingertips can translate object in $17 \mathrm{~mm}$ and rotate object in 36 degrees. The average experimental translation range is $14 \mathrm{~mm}$ and rotation angle is 28 degrees, which is $17 \%$ and $22 \%$ less than the simulation results, respectively. The proposed simulation model of the dual purpose fingertip gives satisfactory results on estimating the gripper manipulation capability of a square object. The model can be improved by adding the initial rotation angle condition and considering the deformation of the inflated air cavities. A further extension to this simulation would involve the addition of object shape and material. This would allow the simulation to predict the motion of the object precisely.

\section{CONCLUSION}

This work proposed and evaluated a novel dual purpose soft fingertip design, presenting a new way to precisely inhand manipulate soft objects without damaging. This approach is based on enhancing the dexterity of robot hands via soft fingertips with tactile sensing and active shape changing. With active shape changing of the embedded air cavities, the fingertip has proved its ability to in-hand manipulate soft objects prehensilely with pressure feedback control. The proposed simulation model gives an evaluation on the manipulation ability of the enhanced gripper, which can be improved by taking the initial rotation angle and deformation of the fingertips into account. The limited dexterity gripper used in this study only has specific object positions for each grasp configuration when equipped with typical soft fingertips. With the proposed closed-loop control approach and the novel dual purpose fingertips, the object position can be adjusted at each grasp configuration, thus increasing largely the gripper's dexterity. The control algorithm can be developed further in future works for more complex prehensile in-hand manipulations that combine translation and rotation with irregular objects. Moreover, the performance of the dual purpose fingertip could be improved to sense force via the inflated air cavities as well.

\section{REFERENCES}

[1] O. M. Andrychowicz, B. Baker, M. Chociej, R. Jozefowicz, B. McGrew, J. Pachocki, A. Petron, M. Plappert, G. Powell, A. Ray et al., "Learning dexterous in-hand manipulation," The International Journal of Robotics Research, vol. 39, no. 1, pp. 3-20, 2020.

[2] N. C. Dafle, A. Rodriguez, R. Paolini, B. Tang, S. S. Srinivasa, M. Erdmann, M. T. Mason, I. Lundberg, H. Staab, and T. Fuhlbrigge, "Extrinsic dexterity: In-hand manipulation with external forces," in 2014 IEEE International Conference on Robotics and Automation (ICRA). IEEE, 2014, pp. 1578-1585.

[3] R. R. Ma, N. Rojas, and A. M. Dollar, "Spherical hands: Toward underactuated, in-hand manipulation invariant to object size and grasp location," Journal of Mechanisms and Robotics, vol. 8, no. 6, 2016.

[4] K. Telegenov, Y. Tlegenov, and A. Shintemirov, "An underactuated adaptive 3d printed robotic gripper," in 2014 10th France-Japan/8th Europe-Asia Congress on Mecatronics (MECATRONICS2014-Tokyo). IEEE, 2014, pp. 110-115.

[5] B. Ward-Cherrier, N. Rojas, and N. F. Lepora, "Model-free precise inhand manipulation with a 3d-printed tactile gripper," IEEE Robotics and Automation Letters, vol. 2, no. 4, pp. 2056-2063, 2017.

[6] M. Ciocarlie, A. Miller, and P. Allen, "Grasp analysis using deformable fingers," in 2005 IEEE/RSJ International Conference on Intelligent Robots and Systems. IEEE, 2005, pp. 4122-4128.

[7] J. R. Amend, E. Brown, N. Rodenberg, H. M. Jaeger, and H. Lipson, "A positive pressure universal gripper based on the jamming of granular material," IEEE Transactions on Robotics, vol. 28, no. 2, pp. 341-350, 2012.

[8] R. Maruyama, T. Watanabe, and M. Uchida, "Delicate grasping by robotic gripper with incompressible fluid-based deformable fingertips," in IEEE/RSJ International Conference on Intelligent Robots and Systems. IEEE, 2013, pp. 5469-5474.

[9] M. Manti, T. Hassan, G. Passetti, N. D'Elia, C. Laschi, and M. Cianchetti, "A bioinspired soft robotic gripper for adaptable and effective grasping," Soft Robotics, vol. 2, no. 3, pp. 107-116, 2015.

[10] Q. Lu, B. A. Clark, M. Shen, and N. Rojas, "An origami-inspired variable friction surface for increasing the dexterity of robotic grippers," IEEE Robotics and Automation Letters, 2020.

[11] A. J. Spiers, B. Calli, and A. M. Dollar, "Variable-friction finger surfaces to enable within-hand manipulation via gripping and sliding," IEEE Robotics and Automation Letters, vol. 3, no. 4, pp. 4116-4123, 2018.

[12] M. K. Johnson and E. H. Adelson, "Retrographic sensing for the measurement of surface texture and shape," in 2009 IEEE Conference on Computer Vision and Pattern Recognition. IEEE, 2009, pp. 1070-1077.

[13] Q. Lu and N. Rojas, "On soft fingertips for in-hand manipulation: Modeling and implications for robot hand design," IEEE Robotics and Automation Letters, vol. 4, no. 3, pp. 2471-2478, 2019.

[14] B.-H. Kim, "Motion analysis of soft-fingertip manipulation tasks," International Journal of Control, Automation, and Systems, vol. 2, no. 2, pp. 228-237, 2004.

[15] N. Rojas, R. R. Ma, and A. M. Dollar, "The gr2 gripper: an underactuated hand for open-loop in-hand planar manipulation," IEEE Transactions on Robotics, vol. 32, no. 3, pp. 763-770, 2016.

[16] L. He, Q. Lu, S. A. Abad Guaman, N. Rojas, and T. Nanayakkara, "Soft fingertips with tactile sensing and active deformation for robust grasping of delicate objects," IEEE Robotics and Automation Letters, 2020. 